ORIGINAL

\title{
Single-amino acid mutation 66SR in Gag-matrix enhances viral single-cycle infectivity of R5-tropic HIV-1rmt
}

\author{
Naoya Doi, Yosuke Sakai, Yasuyuki Miyazaki, Akio Adachi, and Masako Nomaguchi \\ Department of Microbiology, Institute of Biomedical Sciences, Tokushima University Graduate School, Tokushima, Japan
}

\begin{abstract}
We recently constructed two rhesus macaque-tropic human immunodeficiency virus type 1 (HIV-1 rmt) clones with CXCR4 or CCR5 tropism, but a CCR5-tropic HIV-1rmt clone grew more poorly than a CXCR4topic clone. It has been demonstrated that interaction between viral Gag-matrix (MA) and Env-gp41 cytoplasmic tail is important for virion-incorporation of Env. Concordantly, Gag-MA mutations (62QR and 66SR) that rescue defects in virion -incorporation of Env/viral replication were reported. In this study, we analyzed effects of these Gag-MA mutations on R5-tropic HIV-1rmt replication potentials. While introduction of $62 \mathrm{QR}$ into three HIV1rmt clones tested reduced their multi-cycle replication ability in rhesus lymphocytes or abolish single-cycle infectivity for luciferase reporter cells, three R5-tropic HIV-1rmt clones carrying 66SR exhibited similar growth kinetics to those of their parental clones. One such clone, $66 \mathrm{SR}+5 \mathrm{gtu}$, appeared to induce stronger cytopathic effects than parental clone 5gtu. We therefore investigated effects of 66SR mutation on viral replication in more detail. Single-cycle infectivity of $66 \mathrm{SR}+5 \mathrm{gtu}$ was enhanced relative to that of $5 \mathrm{gtu}$, but $66 \mathrm{SR}+5 \mathrm{gtu}$ virion production was significantly decreased compared to the $5 \mathrm{gtu}$ level. Gag-MA 66SR mutation may be useful to improve growth potentials of the R5-tropic HIV-1rmt clones. J. Med. Invest. 62 : 228-232, August, 2015
\end{abstract}

Keywords : HIV-1rmt, Gag-MA, Envelope, R5-tropic

\section{INTRODUCTION}

Human immunodeficiency virus type 1 (HIV-1) envelope glycoprotein (Env) displays a key function in viral life cycle by mediating the entry into host cells $(1,2)$. HIV-1 Env is synthesized as a single polyprotein gp160 and subsequently cleaved to generate gp120 and gp41. Env acts as a trimer composed of a heterodimer of non-covalently linked gp120 and gp41. Gp120 is a surface protein of virions, and binds to receptor CD4 and coreceptor CCR5 or CXCR4. Depending on the usage of CCR5 or CXCR4 for viral entry, HIV-1 strains are categorized into R5-tropic or X4-tropic viruses, respectively. $\mathrm{Gp} 41$ is a transmembrane protein harboring cytoplasmic tail (CT) region, and works for viral-host membrane fusion. Accumulating evidence suggests that the number of Env trimers required for viral entry is different among HIV-1 strains (3-5). Env has a high capacity of mutation to escape from recognition by host immune system, and is a highly diverse protein in HIV-1 proteins. This high mutation rate/diversity of Env induces strain-specific changes for viral fitness, neutralizing antibody resistance, and inhibitor sensitivity, and strongly affects viral phenotypes $(6,7)$.

HIV-1 virion assembly mainly proceeds by cooperation of four major domains of Gag polyprotein, matrix (MA), capsid (CA), nucleocapsid (NC), and p6. Gag-MA is associated with trafficking of the Gag polyprotein to plasma membrane, main site for virion assembly. Gag-CA induces Gag-Gag interaction to assemble viral structural proteins. Gag-NC binds to viral genomic RNA, and Gagp6 is implicated in membrane fission of viral particles and host cells $(1,8)$. It has been demonstrated that Gag-MA also functions for virion-incorporation of Env through interaction with Env gp41

Received for publication May 28, 2015 ; accepted July 10, 2015.

Address correspondence and reprint requests to Akio Adachi or Masako Nomaguchi, Tokushima University Graduate School, 3-18-15 Kuramoto, Tokushima 770-8503, Japan and Fax : +81-88-633-7080.
CT $(1,9)$. Recent study showed that Gag-MA mutations (62QR and 66SR) rescue defects in virion-incorporation of Env/viral replication caused by Gag-MA mutations or by gp41 CT deletion (10). However, effects of these Gag-MA mutations on virion-incorporation of Env/viral replication were examined only for X4-tropic HIV1 clone NL4-3 but not for the others.

We have been working on construction and characterization of rhesus macaque-tropic HIV-1 (HIV-1rmt) clones to establish HIV1/AIDS primate models that would greatly contribute to basic and clinical studies. We have successfully generated HIV-1rmt clones for the first time that overcome species-specific barriers imposed by host restriction factors, APOBEC 3 proteins, TRIM5 proteins, and tetherin (11). Although R5-tropic HIV-1rmt is important for clinical studies on AIDS due to its role in viral persistence and transmission in individuals, our R5-tropic HIV-1rmt clone designated MN5/LSDQgtu (5gtu) grew more poorly than an X4-tropic HIV-1rmt clone designated MN4/LSDQgtu $(11,12)$. In this study, we investigated effects of Gag-MA mutations (62QR and 66SR) described above on R5-tropic HIV-1rmt life cycle (single-cycle infectivity, viron production, and multi-cycle replication). Our results here show that the Gag-MA 66SR mutation positively acts on singlecycle viral infectivity.

\section{MATERIALS AND METHODS}

\section{Plasmid DNAs}

Generation and characterization of HIV-1rmt clones MN4/ LSDQgtu (4gtu) and 5gtu (Fig. 1) have been previously described $(11,12)$. Introduction of Gag-MA $62 \mathrm{QR}$ and 66SR into proviral clones were carried out by the QuickChange site-directed mutagenesis kit (Agilent Technologies Inc.).

Cells, virus preparation, and reverse transcriptase $(R T)$ assay

A reporter cell line TZM-bl carrying a luciferase gene driven by 


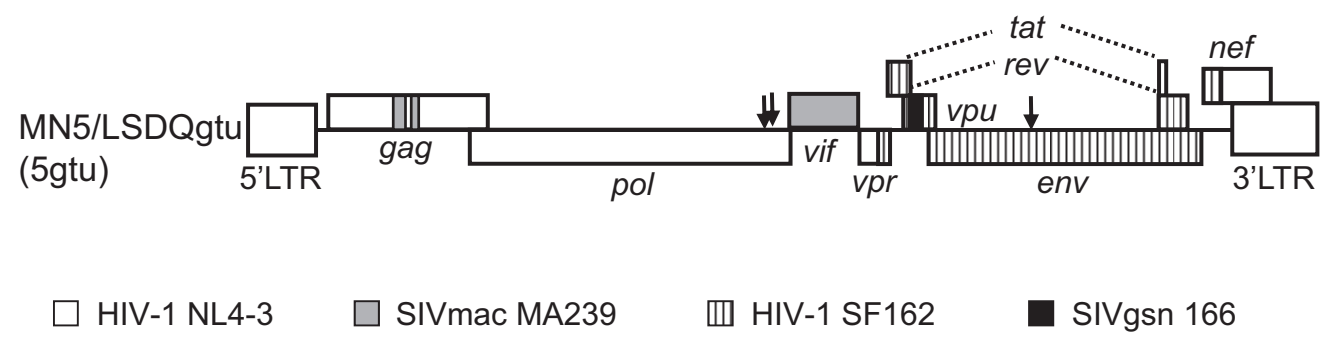

Figure 1 Proviral genome structure of R5-tropic HIV-1rmt MN5/LSDQgtu (5gtu). HIV-1 NL4-3 (15) (GenBank accession number AF324493), SIVmac239 MA239 (19) (GenBank accession number M33262), HIV-1 SF162 (GenBank accession number M65024), and SIVgsn166 (GenBank accession number AF468659) sequences are indicated by white, gray, stripe, and black areas, respectively. Arrows represent the sites of growthenhancing adaptive mutations (18). Clone 5gtu carries four single-amino acid mutations around the two gray areas in the Gag-CA region (11, 20).

viral long-terminal repeat and a human 293T cell line (13) were maintained in Eagle's minimal essential containing $10 \%$ heatinactivated fetal bovine serum. A immortalized lymphocyte cell line M1.3S from a rhesus macaque (14) was maintained in RPMI1640 medium containing $10 \%$ heat-inactivated fetal bovine serum and 50 units/ $\mathrm{ml}$ of recombinant human interleukin-2 (Bio-Rad Laboratories Inc.). Virus stocks for infection experiments were prepared from 293T cells transfected with various proviral clones by the calcium-phosphate co-precipitation method (15), and their virionassociated RT activities were determined as previously described $(11,16)$.

\section{Viral infection experiments}

Multi-cycle replication analysis-M1.3S cells were infected by virus samples with equal RT units, and virus growth was monitored at intervals by RT activity in the culture supernatants. Single-cycle infectivity analysis - Culture supernatants were obtained from the M1.3S infection experiment, and equal RT units $\left(1 \times 10^{4}\right.$ to $\left.2 \times 10^{4}\right)$ of viruses for each sampling day were inoculated into TZM-bl cells $\left(5 \times 10^{3}\right)$. On day 1 post-infection, cell lysates were prepared for luciferase assays (Promega Corporation). Significance relative to 5 gtu was calculated by the Student $t$ test.

\section{Analysis of virion production}

Proviral clones were transfected into 293T cells by the calciumphosphate co-precipitation method as above. Culture supernatants were collected on day 2 post-transfection, and virion production level was determined by RT assays. Significance relative to $5 \mathrm{gtu}$ was calculated by the Student $t$ test.

\section{RESULTS AND DISCUSSION}

Mature infectious HIV-1 virions are generated following appropriate assembly/cleavage of viral Env and Gag precursors (Fig. 2) in the last viral replication phase. Gag-MA plays a pivotal role for the virion-incorporation of Env, and mutations in the region greatly influence the process. It has been reported that HIV-1 Gag-MA $62 \mathrm{QR}$ and 66SR mutations similarly restore defects in virion-incorporation of Env/viral replication displayed by Gag-MA mutant viruses (10). However, introduction of $62 \mathrm{QR}$ or $66 \mathrm{SR}$ into a normal wild-type clone did not affect much the viral growth ability (10). Since these results were obtained from virological analysis of X4tropic HIV-1 NL4-3 clone, we examined the amino acid residues at 62 and 66 sites of various R5-tropic viruses (AD8, JRFL, and YU-2). Residue $62 \mathrm{Q}$ was completely conserved among these viral clones including NL4-3, but residue 66A was found only for JRFL clone (Fig. 3). We further investigated amino acid frequencies at these sites within a large HIV-1 population by the HIV-1/SIVcpz complete genomes (199 strains) in the HIV-1 sequence compendium 2014 (Los Alamos National Laboratory; http : //www.hiv. lanl.gov). Again, $62 \mathrm{Q}$ was very highly conserved, but $66 \mathrm{~S}$ was not much shared by viruses (Table 1). It is well-established that interaction between Gag-MA and Env gp41 CT is critical for virionincorporation of Env and viral replication ability $(1,9,10)$. We therefore compared amino acid sequences in gp $41 \mathrm{CT}$ region of various HIV-1 clones (X4-tropic NL4-3, and R5-tropic SF162, AD8, JRFL, and YU-2 clones). Amino acid sequences in the region were considerably different, and particularly significant sequence characteristics were not noted for NL4-3 clone (Fig. 4).

R5-tropic HIV-1rmt $5 \mathrm{gtu}$ was generated by modification of $\mathrm{gag}$ $\mathrm{CA}$, vif, and $v p u$ genes, and has an env gene derived from HIV-1

Env gp160

\begin{tabular}{|c|c|}
\hline gp120 & gp41 \\
\hline \multicolumn{1}{|c|}{${ }^{\prime}{ }_{\text {gp41 CT }}$} \\
\hline
\end{tabular}

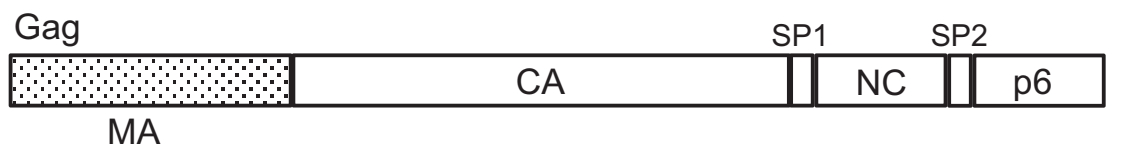

Figure 2 Precursor structures of HIV-1 Env and Gag proteins. Upper : Env subunits gp120 and gp41 are presented. Gp41 cytoplasmic tail (CT) is shown by gray area as indicated. Lower : Four domains and spacer peptide (SP) regions of Gag polyprotein are shown. Dotted area indicates Gag-matrix (Gag-MA) region. CA, capsid ; NC, nucleocapsid. 


\begin{tabular}{|c|c|c|c|}
\hline $\mathrm{NL} 4-3$ & 1 & GARASVLSGGELDKWEKIRLRPGGKKQYKLKH IVWASRELERFAVNPGLLETSEGCRQIL & 60 \\
\hline AD8 & 1 & 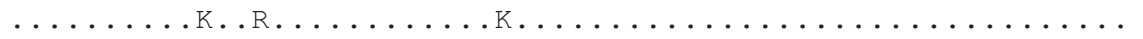 & 60 \\
\hline JRFL & 1 & 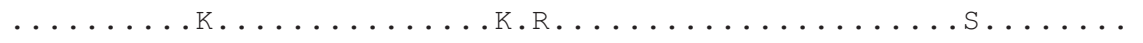 & 60 \\
\hline $\mathrm{YU}-2$ & 1 & $\ldots \ldots$. . . . . . . . . . . . . . . . . . . & 60 \\
\hline $\mathrm{NL} 4-3$ & 61 & GQLLPS $\stackrel{\downarrow}{\downarrow}$ LQTGSEELRSLYNT I AVLYCVHQRI DVKDTKEALDKIEEEQNKSKKKAQQAAAD & \\
\hline AD8 & 61 & 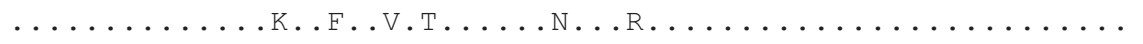 & \\
\hline JREL & 61 & 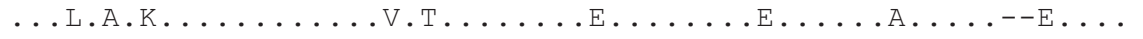 & \\
\hline $\mathrm{YU}-2$ & 61 & $\ldots \ldots \ldots \ldots \ldots \ldots$. $\ldots \ldots \ldots$. $\ldots$. $\ldots \ldots \ldots \ldots \ldots \ldots \ldots$ & 20 \\
\hline NL4-3 & 121 & TGNNSQVSQNY & \\
\hline AD 8 & 121 & AEKS ..... & \\
\hline $\mathrm{L}$ & 119 & $\ldots s \ldots \ldots$ & \\
\hline $\mathrm{YU}-2$ & 121 & $\ldots s \ldots . .$. & 31 \\
\hline
\end{tabular}

Figure 3 Amino acid sequence alignment of Gag-MA proteins from various HIV-1 strains. Sequences were aligned by Genetyx Ver. 11. Gag start residue $\mathrm{M}$ (Met) is removed from the sequences, and second residue G (Gly) is presented at amino acid position 1. Arrows show the amino acid positions 62 and 66 .

Table 1 Amino acid frequency at positions 62 and 66 in Gag-MA

\begin{tabular}{lcrr}
\hline Positions & Amino acid & No. & $\%$ \\
\hline 62 & Q & 197 & 99.0 \\
& H & 1 & 0.5 \\
& K & 1 & 0.5 \\
66 & S & 69 & 34.7 \\
& A & 101 & 50.8 \\
& T & 26 & 13.1 \\
& D & 1 & 0.5 \\
& N & 1 & 0.5 \\
& $X^{\mathrm{b}}$ & 1 & 0.5 \\
\hline
\end{tabular}

a Data are from 199 sequences of HIV-1/SIVcpz complete genomes in the HIV Sequence Compendium, 2014 (Los Alamos National Laboratory ; http : //www.hiv.lanl.gov).

b Unidentified amino acid
SF162 (Fig. 1). To examine effects of Gag-MA mutations on 5gtu, we introduced $62 \mathrm{QR}$ and $66 \mathrm{SR}$ into $5 \mathrm{gtu}$ to construct HIV-1rmt clones $62 \mathrm{QR}+5 \mathrm{gtu}$ and $66 \mathrm{SR}+5 \mathrm{gtu}$, respectively. Viruses were prepared from 293T cells transfected with proviral clones (5gtu, 62QR+ $5 \mathrm{gtu}$, and $66 \mathrm{SR}+5 \mathrm{gtu}$ ), and inoculated into rhesus lymphocyte M1.3S cells. As shown in Fig. 5, while $62 \mathrm{QR}+5$ gtu grew significantly more poorly than $5 \mathrm{gtu}, 66 \mathrm{SR}+5 \mathrm{gtu}$ exhibited slightly more retarded growth kinetics relative to $5 \mathrm{gtu}$. To see if this result is applicable to the other R5-tropic viruses, and to confirm a generalization of above observation, we generated two other R5-tropic HIV-1rmt clones carrying an env gene from a simian immunodeficiency virus/HIV-1 chimeric virus clone (SHIV $\left.\mathrm{ADB}_{-\mathrm{EH}}\right)$ (17) or from an HIV-1 clinical isolate. We then performed the M1.3S infection experiments as above. Introduction of $62 \mathrm{QR}$ into these two viral clones eliminated the viral replication ability, but that of 66SR did not show a significant effect on their growth potentials relative to parental clones (data not shown). Totally, $62 \mathrm{QR}$ mutation was detrimental to R5-tropic viruses but not 66SR. In agreement with the previous data for X4-tropic NL4-3 (10), the 62QR mutation severely impeded viral single-cycle infectivity of our there R5tropic viruses tested here for TZM-bl cells (data not shown). This result is consistent with the conserved nature of Gag-MA 62Q in

\begin{tabular}{|c|c|c|c|}
\hline 162 & 1 & VNRVRQGYSPLSFQTRFPAPRGPDRPEGIEEEGGERDRDRSSPLVHGLLALIWDDLRSLC & 60 \\
\hline D8 & 1 & $\ldots \ldots \ldots \ldots \ldots$ HL $\ldots \ldots \ldots \ldots$. . . . . . . . F . F . . . . & 60 \\
\hline REL & 1 & 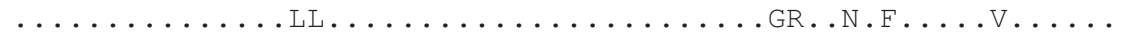 & 60 \\
\hline J2 & 1 & $\ldots \ldots \ldots \ldots$. & 60 \\
\hline $4-3$ & 1 & 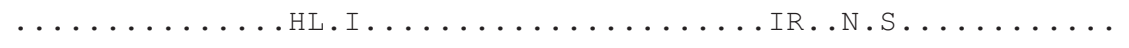 & 60 \\
\hline 162 & 61 & LFSYHRLRDL I LIAARIVE LLGRRGWEALKYWGNLLQYWIQELKNSAVSLFDAIAIAVAE & 120 \\
\hline 8 & 61 & 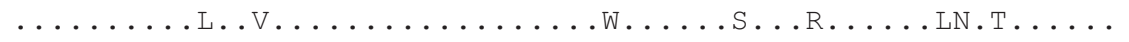 & 0 \\
\hline REL & 61 & $\ldots \ldots \ldots$. . . & 0 \\
\hline J2 & 61 & $\ldots \ldots \ldots$. $\ldots$ VT $\ldots \ldots \ldots \ldots$ GV $\ldots$. $\ldots \ldots \ldots \ldots \ldots$ LN.$\ldots \ldots$ & 20 \\
\hline $4-3$ & 61 & 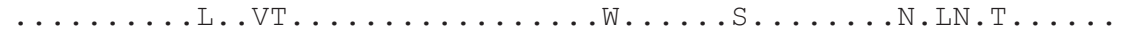 & 0 \\
\hline 162 & 121 & GTDRI IEVAQRI GRAFLHIPRRIRQGFERALL & \\
\hline 88 & 121 & 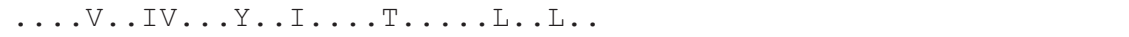 & 2 \\
\hline $\mathrm{FL}$ & 121 & 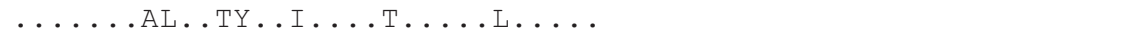 & 2 \\
\hline & 121 & 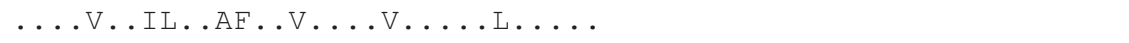 & \\
\hline $4-3$ & 121 & 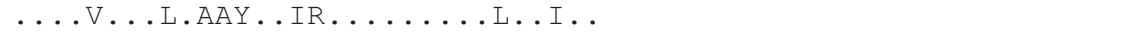 & \\
\hline
\end{tabular}

Figure 4 Amino acid sequence alignment of gp41 CT from various HIV-1 strains. Sequences were aligned by Genetyx Ver. 11. The gp41 CT sequence of 5gtu is identical to that of HIV-1 SF162. 


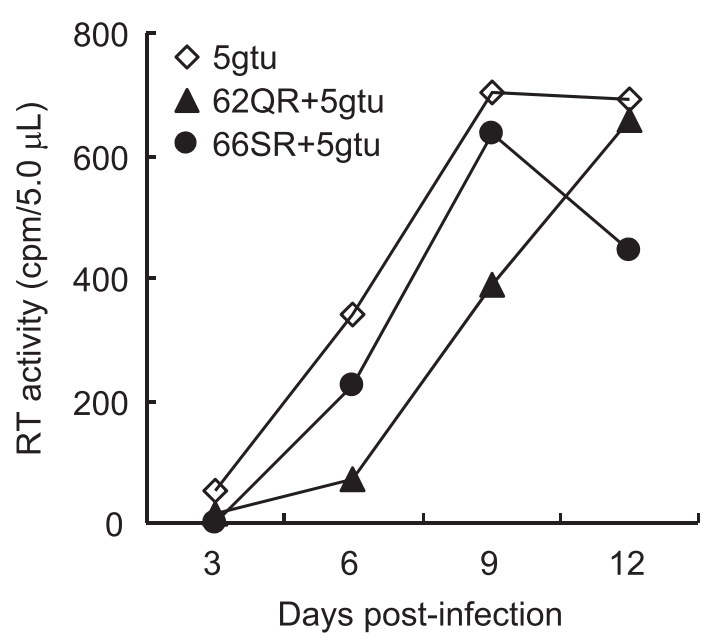

Figure 5 Growth kinetics in rhesus macaque M1.3S cells of various HIV-1rmt clones. Input viruses were prepared from 293T cells transfected with proviral clones indicated, and inoculated into M1.3S cells. For infection, $2.0 \times 10^{5} \mathrm{M} 1.3 \mathrm{~S}$ cells were inoculated with equal viral RT units $\left(1.0 \times 10^{6}\right)$. Virus growth was monitored at intervals by RT activity in the culture supernatants.

an HIV-1 population (Fig. 3 and Table 1).

We noticed that $66 \mathrm{SR}+5 \mathrm{gtu}$ appeared to induce stronger cytopathic effects than $5 \mathrm{gtu}$ despite their considerably similar growth kinetics in M1.3S cells. To further examine biological properties of $5 \mathrm{gtu}$ and $66 \mathrm{SR}+5 \mathrm{gtu}$, we first determined multi-cycle growth kinetics in M1.3S cells of three HIV-1rmt clones (X4-tropic 4gtu, R5-tropic 5gtu, and R5-tropic 66SR+5gtu). Major difference between $4 \mathrm{gtu}$ and $5 \mathrm{gtu}$ is their env sequences : 4gtu env from HIV-1 NL4-3 and 5gtu env from SF162, respectively, and both have some growth-enhancing mutations in the env gene $(11,12)$. Input viruses obtained from transfected 293T cells were inoculated into M1.3 cells, and virus replication was periodically monitored. As shown in Fig. 6A, 4gtu grew better than 5 gtu (note the peak day for RT production), consistent with a previous report (12). Virus growth kinetics of $5 \mathrm{gtu}$ and $66 \mathrm{SR}+5 \mathrm{gtu}$ were similar, but virion production levels on the peak day (day 15 post-infection) and thereafter, as judged by RT activity, of 66SR+5gtu were lower than those of $5 \mathrm{gtu}$. We were interested in determining the efficiency of early and late replication phases of the three HIV-1rmt clones. To this end, viral single-cycle infectivity for TZM-bl cells (early) of progeny virions produced from infected M1.3S cells and progeny virion level (late) upon transfection into 293T cells were determined. Culture supernatants were collected from infected M1.3S cells (Fig. 6A), and equal RT units of viruses from each sampling day were inoculated into TZM-bl cells. On day 1 post-infection, cell lysates were prepared for luciferase assays. Single-cycle viral infectivity was calculated as relative luciferase activity to that of 5gtu. In Fig. 6B, relative infectivity was presented as the average of infectivity for each day (from day 6 to 33). Viral infectivity of 4gtu and 66SR+5gtu was higher than that of $5 \mathrm{gtu}$, and $66 \mathrm{SR}+5 \mathrm{gtu}$ showed comparable infectivity to $4 \mathrm{gtu}$. We next examined virion production level of the three HIV-1rmt clones in transfected 293T cells. In fragile macaque M1.3S cells, it is very difficult to obtain constant and reproducible results by transfection. As shown in Fig. 6C, while progeny virions of 4gtu and 5gtu were at similar levels, 66SR+5gtu produced those at a level reduced to around $40 \%$ to that of $5 \mathrm{gtu}$. Gag-MA is demonstrated to fulfill its function at various steps in the HIV-1 assembly process $(1,8,9)$. It is therefore conceivable that $66 \mathrm{SR}$ mutation in Gag-MA may negatively affect this stage. Collectively, our results on the 66SR mutant virus showed that introduction of
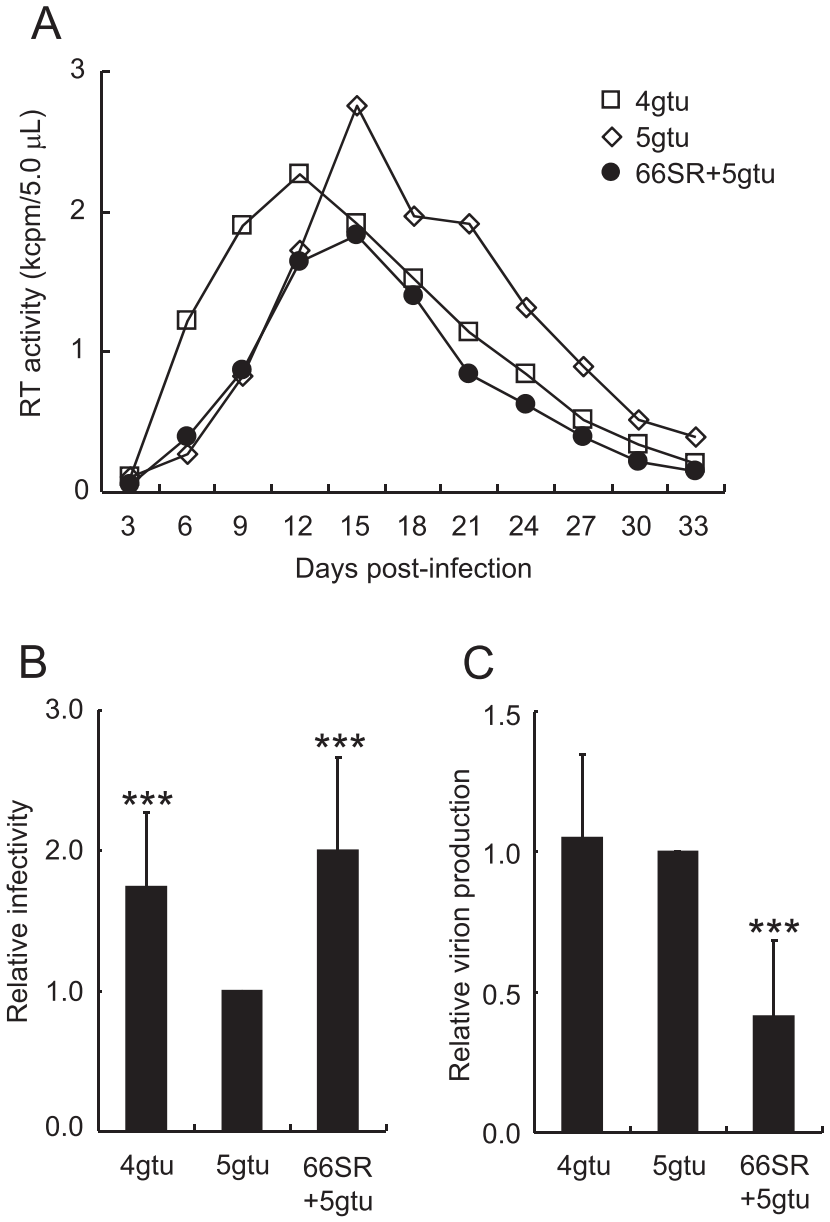

Figure 6 Replication properties of various HIV-1rmt clones. (A) Multicycle replication kinetics in rhesus lymphocyte M1.3S cells. Input viruses were prepared from 293T cells transfected with proviral clones indicated, and inoculated into M1.3S cells. For infection, $1.0 \times 10^{6} \mathrm{M} 1.3 \mathrm{~S}$ cells were inoculated with equal viral RT units $\left(6.6 \times 10^{5}\right)$. Virus growth was monitored at intervals by RT activity in the culture supernatants. (B) Viral single-cycle infectivity. Culture supernatants obtained from the M1.3S infection experiment in (A) were used for determination of viral infectivity here. Equal RT units of virus samples from each day (from day 6 to 33 in A) were inoculated into TZM-bl cells, and on day 1 post-inoculation, cell lysates were prepared for luciferase assays. Infectivity is calculated as luciferase activity relative to that exhibited by $5 \mathrm{gtu}$ for each sampling day. Mean values of relative infectivity obtained from samples of day 6 to 33 are shown with the standard deviations (SD). Significance relative to $5 \mathrm{gtu}$ as calculated by the Student $t$ test is shown $\left({ }^{\star \star \star}, P<0.01\right)$. (C) Virion production. Proviral clones indicated were transfected into 293T cells, and on day 2 post-transfection, progeny virus production was measured by RT activity in the culture supernatants. RT activity relative to that produced by $5 \mathrm{gtu}$ is presented. Mean values \pm SD from at least five independent experiments are shown. Significance relative to $5 \mathrm{gtu}$ as calculated by the Student $t$ test is shown ( $\left.{ }^{\star \star}, P<0.01\right)$.

66SR into 5gtu gives an affirmative effect on early replication phase (single-cycle viral infectivity in Fig. 6B) but a contradictious result on late phase (virion production level in Fig. 6C), generating similar growth kinetics in multi-cycle infection experiments (Figs. 5 and $6 \mathrm{~A})$. Lower virus level observed for $66 \mathrm{SR}+5 \mathrm{gtu}$ relative to that for parental $5 g t u$ (Figs. 5 and 6A) may be explained by its feeble ability to produce progeny virions (Fig. 6C), and also, at least in part, by its cytopathogenic property described above. Quite different from our results, positive effects of the 66SR mutation on viral single-cycle infectivity were not observed for HIV-1 NL4-3 clone (10). This may be attributable to differences in gp41 CT amino acid 
sequences (Fig. 4). It would be plausible to assume that Gag-MA 66SR have appreciable impacts on viral clones carrying some specific Env sequences.

In conclusion, we demonstrate here that Gag-MA 66SR mutation increases the single-cycle infectivity of R5-tropic HIV-1rmt 5 tgu but impedes the virion production efficiency. Because mutations in Gag-MA affect much the virion assembly process as described above, this defect may be restored by combinations of the other Gag-MA mutations previously reported (10). Improvement of HIV-1rmt clones carrying 66SR in the virion production level may lead to generation of new viral clones with better growth potentials. In this regard, we already gained a number of growthenhanced viral clones by long-term cultures of virus-infected cells (18). Adaptation of $66 \mathrm{SR}+5 \mathrm{gtu}$ to M1.3S cells in a similar way is in progress. These experimental studies would provide technically useful information to improve growth potentials of R5-tropic HIV$1 \mathrm{rmt}$ clones, and contribute to the establishment of HIV-1/AIDS primate models. Obtaining R5-tropic HIV-1rmt clones pathogenic for rhesus macaques represents the cornerstone of AIDS models, and may help the development of clinical approaches aimed at better treatments of infected individuals.

\section{ACKNOWLEDGEMENTS}

We thank Kazuko Yoshida for editorial assistance. We are indebted to Drs. Masashi Shingai and Malcolm A. Martin (NIAID, $\mathrm{NIH}$, USA.) for their generous gift of $\mathrm{pSHIV}_{\mathrm{AD} 8 \text {-EH }}$ proviral clone. TZM-bl cells were obtained from the NIH AIDS Reagent Program. This research was supported in part by a grant from Japan Agency for Medical Research and Development, AMED.

\section{CONFLICT OF INTEREST}

The authors declare that no competing interests exist.

\section{REFERENCES}

1. Checkley MA, Luttge BG, Freed EO : HIV-1 envelope glycoprotein biosynthesis, trafficking, and incorporation. J Mol Biol 410 : 582-608, 2011

2. Wilen CB, Tilton JC, Doms RW : HIV : cell binding and entry. Cold Spring Harb Perspect Med $2:$ 8, 2012

3. Brandenberg OF, Magnus C, Rusert P, Regoes RR, Trkola A : Different infectivity of HIV-1 strains is linked to number of envelope trimers required for entry. PLoS Pathog 11 : e1004595, 2015

4. Klasse PJ : Modeling how many envelope glycoprotein trimers per virion participate in human immunodeficiency virus infectivity and its neutralization by antibody. Virology $369: 245$ 262, 2007

5. Magnus C, Rusert P, Bonhoeffer S, Trkola A, Regoes RR : Estimating the stoichiometry of human immunodeficiency virus entry. J Virol 83 : 1523-1531, 2009

6. Araújo LA, Almeida SE : HIV-1 diversity in the envelope glycoproteins : implications for viral entry inhibition. Viruses 5 :
595-604, 2013

7. Burton DR, Mascola JR: Antibody responses to envelope glycoproteins in HIV-1 infection. Nat Immunol 16 : 571-576, 2015

8. Sundquist WI, Kräusslich HG : HIV-1 assembly, budding, and maturation. Cold Spring Harb Perspect Med 2 : a006924, 2012

9. Tedbury PR, Freed EO : The role of matrix in HIV-1 envelope glycoprotein incorporation. Trends Microbiol 22 : 372-378, 2014

10. Tedbury PR, Ablan SD, Freed EO : Global rescue of defects in HIV-1 envelope glycoprotein incorporation : implications for matrix structure. PLoS Pathog 9 : e1003739, 2013

11. Nomaguchi M, Yokoyama M, Kono K, Nakayama EE, Shioda T, Doi N, Fujiwara S, Saito A, Akari H, Miyakawa K, Ryo A, Ode H, Iwatani Y, Miura T, Igarashi T, Sato H, Adachi A : Generation of rhesus macaque-tropic HIV-1 clones that are resistant to major anti-HIV-1 restriction factors. J Virol 87 : 11447-11461, 2013

12. Doi N, Okubo A, Yamane M, Sakai Y, Adachi A, Nomaguchi $\mathrm{M}$ : Growth potentials of CCR5-tropic/CXCR4-tropic HIV-1mt clones in macaque cells. Front Microbiol $4: 218,2013$

13. Lebkowski JS, Clancy S, Calos MP : Simian virus 40 replication in adenovirus-transformed human cells antagonizes gene expression. Nature $317:$ 169-171, 1985.

14. Doi N, Fujiwara S, Adachi A, Nomaguchi M : Rhesus M1.3S cells suitable for biological evaluation of macaque-tropic HIV/ SIV clones. Front Microbiol $2: 115,2011$

15. Adachi A, Gendelman HE, Koenig S, Folks T, Willey R, Rabson A, Martin MA : Production of acquired immunodeficiency syndrome-associated retrovirus in human and nonhuman cells transfected with an infectious molecular clone. J Virol 59 : 184-291, 1986

16. Willey RL, Smith DH, Lasky LA, Theodore TS, Earl PL, Moss B, Capon DJ, Martin MA : In vitro mutagenesis identifies a region within the envelope gene of the human immunodeficiency virus that is critical for infectivity. J Virol $62: 139-147$, 1988

17. Shingai M, Donau OK, Schmidt SD, Gautam R, Plishka RJ, Buckler-White A, Sadjadpour R, Lee WR, LaBranche CC, Montefiori DC, Mascola JR, Nishimura Y, Martin MA : Most rhesus macaques infected with the CCR5-tropic SHIV(AD8) generate cross-reactive antibodies that neutralize multiple HIV-1 strains. Proc Natl Acad Sci U S A 109 : 19769-19774, 2012

18. Nomaguchi M, Doi N, Fujiwara S, Saito A, Akari H, Nakayama EE, Shioda T, Yokoyama M, Sato H, Adachi A: Systemic biological analysis of the mutations in two distinct HIV-1mt genomes occurred during replication in macaque cells. Microbes Infect $15: 319-328,2013$

19. Shibata R, Kawamura M, Sakai H, Hayami M, Ishimoto A, Adachi A : Generation of a chimeric human and simian immunodeficiency virus infectious to monkey peripheral blood mononuclear cells. J Virol 65 : 3514-3520, 1991

20. Nomaguchi M, Yokoyama M, Kono K, Nakayama EE, Shioda T, Saito A, Akari H, Yasutomi Y, Matano T, Sato H, Adachi A : Gag-CA Q110D mutation elicits TRIM5-independent enhancement of HIV-1mt replication in macaque cells. Microbes Infect $15: 56-65,2013$ 